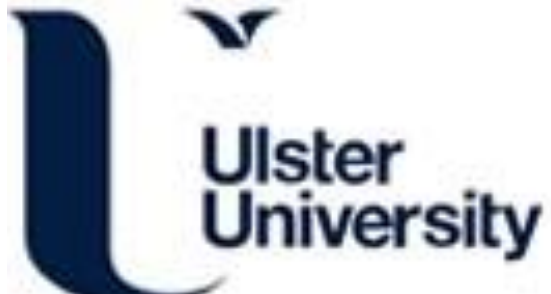

\section{Blood vessel segmentation in retinal images using echo state networks}

Souahlia, A., Belatreche, A., Benyettou, A., \& Curran, K. (2017). Blood vessel segmentation in retinal images using echo state networks. In Unknown Host Publication IEEE. http://uir.ulster.ac.uk/36319/1/4\%20\%20IJCNN\%202016\%20Paper\%20\%2316470\%20Experimental\%20Evaluation\%20of\%20Echo\%20State\%20N etwork.txt

Link to publication record in Ulster University Research Portal

\section{Published in:}

Unknown Host Publication

Publication Status:

Published (in print/issue): 04/02/2017

\section{Document Version \\ Author Accepted version}

\section{General rights}

Copyright for the publications made accessible via Ulster University's Research Portal is retained by the author(s) and / or other copyright owners and it is a condition of accessing these publications that users recognise and abide by the legal requirements associated with these rights.

\section{Take down policy}

The Research Portal is Ulster University's institutional repository that provides access to Ulster's research outputs. Every effort has been made to ensure that content in the Research Portal does not infringe any person's rights, or applicable UK laws. If you discover content in the Research Portal that you believe breaches copyright or violates any law, please contact pure-support@ulster.ac.uk. 


\section{Blood Vessel Segmentation In Retinal Images Using Echo State Networks}

\author{
Abdelkerim Souahlia \\ Faculty of Technology \\ University of Djelfa, \\ Algeria \\ Email: a.souahlia@univ- \\ djelfa.dz
}

\author{
Ammar Belatreche \\ Department of Computer \\ and Information Sciences \\ Northumbria University, UK \\ Email: \\ ammar.belatreche@northumb \\ ria.ac.uk
}

\author{
Abdelkader Benyettou \\ Faculty of Mathematics \\ and Computer Science \\ University of Sciences and \\ Technology, Algeria \\ Email: \\ aek.benyettou@univ- \\ usto.dz
}

\author{
Kevin Curran \\ Intelligent Systems \\ Research Centre \\ Ulster University, UK \\ Email: \\ kj.curran@ulster.ac.uk
}

\begin{abstract}
We propose a novel supervised technique for blood vessel segmentation in retinal images based on echo state networks. Retinal vessel segmentation is widely used for numerous clinical purposes such as the detection of various cardiovascular and ophthalmologic diseases. A large number of retinal vessel segmentation methods have been reported, yet achieving accurate and efficient vessel segmentation still remains a challenge. Recently, reservoir computing has drawn much attention as a new computing framework based on recurrent neural networks. The Echo State Network (ESN), which uses neural nodes as the computing elements of the recurrent network, represents one of the efficient learning models of reservoir computing. This paper investigates the viability of echo state networks for blood vessel segmentation in retinal images. Initial image features are projected onto the echo state network reservoir which maps them, through its internal nodes activations, into a new set of features to be classified into vessel or non-vessel by the echo state network readout which consists, in the proposed approach, of a multi-layer perceptron. Experimental results on the publicly available DRIVE dataset, commonly used in retinal vessel segmentation research, demonstrate the ability of the proposed method in achieving promising performance results in terms of both segmentation accuracy and efficiency.
\end{abstract}

Keywords-echo state network; retinal images; vessel segmentation; pixel classification; feature extraction

\section{INTRODUCTION}

Blood vessel segmentation in retinal images is an important stage in computer based retinal image analysis and diagnosis of retinal diseases. It is useful for numerous clinical purposes such diagnosis, screening and treatment of several pathologies like hypertension, diabetes, cardiovascular and ophthalmologic diseases [1], computer-assisted laser surgery [1] and other retina components identification such as the optic disc and the fovea [2]. It is also useful for temporal registration of retinal images. The integration of information from registered images serves for automatic monitoring of the progression of certain diseases [3]. The vascular tree extracted from a retinal image is also used for biometric identification [4].
With the growing importance of vessel segmentation in retinal images and manual delineation of the vessels being costly in terms of effort and time, automatic accurate and efficient segmentation methods of retinal vessels are needed. Since the early work of Chaudhuri et al. in 1989 [5], a large number of automated methods have been reported in literature. Recent surveys of these methods can be found in [1], [6]-[8]. Based on the training mode, the existing methods can be classified into supervised and unsupervised methods. Usually the supervised methods result in higher segmentation accuracy than their unsupervised counterparts. However, the most reported supervised methods (see Section II) need a long processing time due to the applied pre- and post-processing operations. In this paper, we propose an efficient retinal vessel segmentation method based on ESN. We show that this technique can achieve comparable segmentation accuracy with state-of-the-art methods without using any pre- or postprocessing and outperforms all of them in terms of processing time.

Recently, the concept of reservoir computing (RC) has been used to simplify the training of recurrent neural networks (RNN) [9], [10]. The two main realisations of RC are the Echo State Network, proposed by Herbert Jaeger [11], and the Liquid State Machine proposed by Maass et al. [12]. In this paper, we focus on the former type which consists of a large recurrent neural network whose connection weights are randomly generated and is followed by a readout layer which is trained using commonly known linear regression algorithms. The ESN attracted much attention due to its simple computational model that has been used successfully in many application tasks [13]-[18].

In this paper, we carry out an investigation of the viability of an ESN based framework for blood vessel segmentation in retinal fundus images. An ESN consisting of a reservoir and a simple feedforward artificial neural network has been designed. While the reservoir serves as an image feature extractor of the input image features, the feedforward neural network is used as the readout function of the ESN, which classifies the image features which are captured by the output nodes of the reservoir. The obtained segmentation is thoroughly evaluated using objective segmentation quality 
metrics such as accuracy, specificity and sensitivity. Experiments on the widely used DRIVE (Digital Retinal Images for Vesse Extraction) dataset show promising results and demonstrate that the proposed Echo State Network based approach can achieve competitive segmentation performance in terms of both accuracy as well as efficiency compared with related state-of-the-art methods. To the best of our knowledge, this is the first application of echo state networks for blood vessel segmentation in retinal images.

The rest of the paper is organized as follows: A review of the related work is presented in Section II while Section III presents a description of the ESN concept and its main properties. A description of the proposed ESN based framework and its application to blood vessel segmentation in retinal images is presented in Section IV. Practical choices related to the setup of the proposed framework are reported in Section V, Section VI presents the evaluation experiments conducted on the DRIVE dataset to validate the proposed approach and a comparison of the obtained results with stateof-the-art methods. Section VII concludes the paper and highlights potential future research directions.

\section{RELATED WORK}

Based on the training mode, existing algorithms for blood vessel segmentation in retinal fundus images can be classified into two main classes, namely unsupervised and supervised methods.

\section{A. Unsupervised Methods:}

Unsupervised blood vessel segmentation algorithms can be further grouped into 3 categories (1) matched filtering approaches, (2) vessel tracking approaches and (3) modelbased approaches. Matched filtering techniques search vessel segments using convolution of a 2-D kernel with the retinal image, typically a Gaussian based kernel or its derivatives is used in different directions to detect vessels of different orientations [5], [19]. In a recent work, Azzopardi et al. [20] proposed a technique of vessels detection based on a combination of shifted filter responses (COSFIRE). The matched filtering techniques work well for healthy images but they usually increase the false positives, i.e. the number of non-vessel pixels that are detected as vessels, in pathological images [1]. In vessel tracking methods [21], [22], tracking consists of segmenting vessels by following vessel centrelines using local information after selecting a set of reliable seed points on the vessel tree to use as starting points It is reported that these methods are able to produce accurate vessel widths, however they often fail to detect blood vessels with no seed points [23]. Finally, the model-based group of approaches include the vessel profile models [24] and the deformable models [25]. Profile models consider that the vessel intensities vary according to a certain model such as a Gaussian or a second-order derivative Gaussian [26]. The deformable (a.k.a snake) models is also used for vessel segmentation in retinal images [27]. A snake is defined by as active contour model which uses an iterative adaptation to match the shape of the desired structure. The model-based approaches suffer from the fact that the vessels are usually crossed and having many branches.

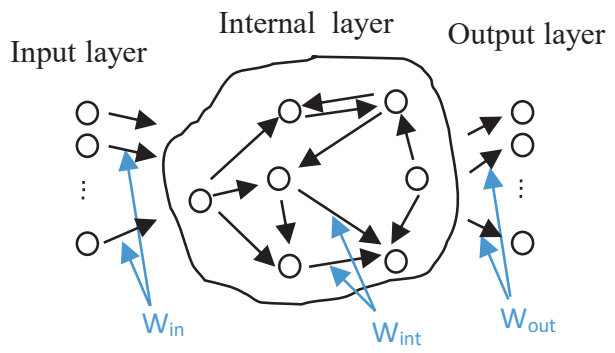

Fig. 1. Generic Echo State Network architecture

\section{B. Supervised Methods:}

Blood vessel segmentation based on supervised methods can be approached as a pixel classification problem where image pixels are represented by a set of features (e.g. spatial, texture, intensity etc.) and a classifier is then trained using the extracted feature vectors from hand-labelled gold standard images to discern vessel pixels from non-vessel ones. Different features such as Gaussian and its derivatives [28], ridge-based vessel detection features [29], Gabor filter responses [30]-[32], moment-invariant [33], grey-level features e.g. mean and standard deviation [32], [33], gradient vector field [31], morphological transformation [31], line strength measures [31], the stroke width transform (SWT) [32], Weber's local descriptors (WLD) [32] and vesselness [32] have been used in vessels segmentation based on supervised techniques. The extracted features are classified using different classifiers such as K-nearest neighbour [28], [29], Gaussian mixture model, Bayesian classifier [30], multilayer feedforward neural network [33], ensemble system of bagged and boosted decision trees [31] and random forest classifier [32]. Recently, Li et al. [23] have reformulated the problem of blood vessel segmentation as a data transformation between retinal images and the vessel map. They have employed a deep neural network for modelling the reformulated transformation task. It is well known that supervised blood vessel segmentation techniques usually result in higher accuracy than their unsupervised counterparts given the a priori knowledge used by the supervised methods (i.e. ground truth pixel labels). However, such ground truth labelled data may not always be available for training these supervised techniques. In addition, as pixel labelling is carried out manually by experts, there is often disagreement between them in identifying blood vessels [1].

\section{Echo StATE NeTWORK}

The general architecture of an ESN as described in [11] is shown in Fig. 1. An ESN consists of three layers: an input layer, a dynamic reservoir which represent the internal layer of the ESN, and read-out layer which represents the output layer of the ESN [11]. The input layer connects to the nodes of the dynamic reservoir through random synaptic

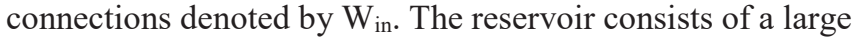
number $(\mathrm{N})$ of randomly generated nodes which are sparsely connected through randomly weighted connections denoted by $\mathrm{W}_{\text {int. }}$ The nodes of the reservoir are connected to the output layer, which contains L output nodes, through output weights denoted by $\mathrm{W}_{\text {out }}$. 


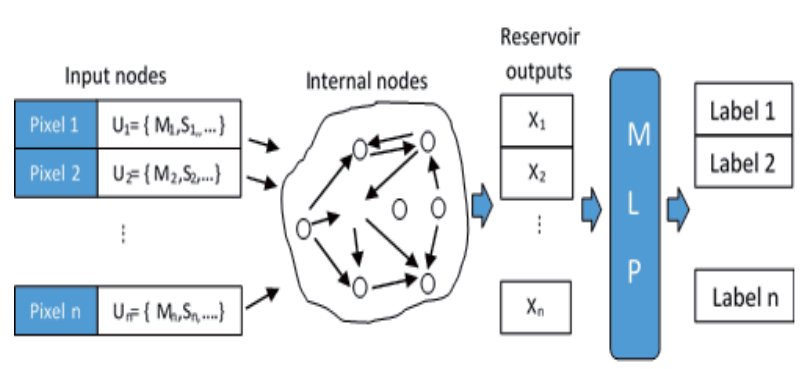

Fig. 2. Proposed ESN framework; Mi, Si represent the mean and standard deviation values of pixel I neighbours. ESN:

The following two equations control the dynamics of the

$$
\begin{gathered}
x(n+1)=f\left(W_{\text {int }} \times x(n)+W_{\text {in }} \times u(n+1)\right) \\
y(n+1)=g\left(W_{\text {out }} x(n+1)\right)
\end{gathered}
$$

where $x(n)$ and $x(n+1)$ represent the reservoir states at times $n$ and $n+1$, respectively. $y(n+1)$ represents the ESN output at time $n+1 . \mathrm{W}_{\text {int }}$ is of size $N x N$ and represents the matrix of connection weights linking the reservoir nodes. $W_{i n}$, of size $K x N$, represents the weights matrix of the input connections. $u(n+1)$ represents the input data at time step $n+1$ which is given by $u(n+1)=\left\{u_{j}(n+1): j=1, \ldots, K\right\} . f$ represents the reservoir nodes activationffunction, usually hyperbolic tangent/sigmoidal functions are used. $W_{\text {out }}$, of size $N \times L$, represents the readout weight matrix. Finally, the function $g$ represents the activation function of the output neurons which linear in general.

The training algorithm of the ESN consists of simply identifying the readout layer weights which minimize the mean squared error between $Y$, the actual, and $Y_{d}$, the desired output, given by:

$$
W_{\text {out }}=\arg _{w}\left(\min \left\|Y-Y_{d}\right\|^{2}\right)
$$

where symbol $\|\cdot\|$ denotes the Euclidean norm and $W_{\text {out }}$ is given by:

$$
W_{\text {out }}=\left(X^{T} X\right)^{-1} X^{T} Y_{d}
$$

$X$ is a matrix which accumulates all of the reservoir states and $X^{T}$ represents its transpose. $\left(X^{T} X\right)^{-1}$ denotes the inverse of the square matrix $X^{T} X$.

The minimisation of the readout layer error is usually done using linear regression algorithms [11]. However, in this work, we have proposed to use a multi-layer perceptron as the ESN readout layer trained by error backpropagation instead of a single-layer trained by linear regression.

\section{The Proposed Blood Vessel Segmentation Using THE ESN-BASED FRAMEWORK}

Unlike the other supervised techniques reviewed in Section II.B, the proposed ESN-based approach projects the extracted pixel features (described in Section V.B) onto a new feature space represented by the reservoir internal activations. The new feature space is represented by the reservoir output matrix. The next step is to train the readout layer, which in our case consists of an MLP, using a set of pixels selected
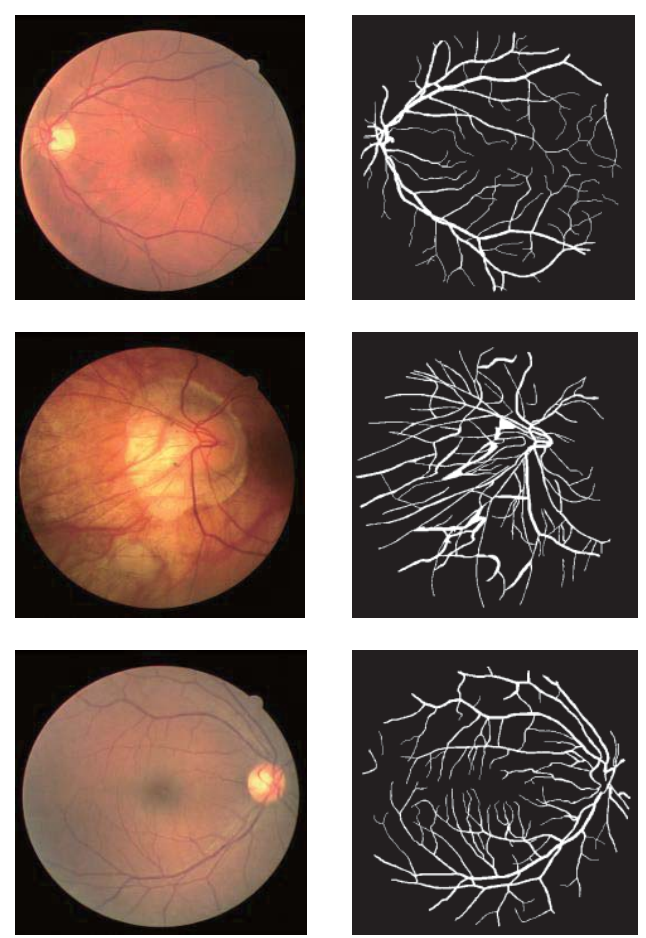

Fig. 3. Examples of retinal images from the DRIVE dataset. Left column: Original images. Right column: corresponding ground truths.

from the training image dataset. The ground-truthsegmentation of the training images is used as the desired outputs. Then, the trained MLP (readout) is used for segmenting the pixels of newly unseen (testing) retinal images into vessel or background. Fig. 2 illustrates the proposed approach.

\section{EXPERIMENTAL SETUP}

\section{A. The DRIVE dataset}

In order to thoroughly evaluate the ESN-based blood vessel segmentation approach, we have conducted extensive experiments on the widely used DRIVE dataset which contains forty retinal images of size $768 \times 584$ pixels each with a circular field of view (FOV) with a diameter of 540 pixels approximately [29]. The DRIVE dataset is split into a training set (20 images) and a test set (20 images) [29]. Fig. 3 shows samples of the original images from the DRIVE dataset with their corresponding ground truths.

\section{B. Retinal image feature extraction}

We have used simple features computed from pixels intensities in addition to other extracted Gabor based texture features and Gradient operator. As the green channel was found to offer better contrast between vessel and background pixels and the red and blue planes were found to be rather noisy, it has been used in the proposed approach to extract all of the RGB retinal image features. [29], [32] and [33]. It is worth noting that the features are extracted without any preprocessing of the images to preserve the vessel structure and especially avoid the risk of thin vessel removal. 


\section{1) Intensity-based features}

For each pixel, we have used the average and the standard deviation of its neighbouring pixels within a 19x19-pixel window. The size of the neighbourhood window has been chosen by trial and error.

\section{2) Gabor-based features}

Gabor filter is a well known linear filter that is widely used in image processing, e.g. edge detection and characterisation of image textures. Due to the structure of vascular tree in retinal images, the Gabor filter has also been used in many works of vessel segmentation [5], [19], [30][32] because of its capability to be tuned to specific frequencies, directions and scales, hence detecting vessels of different orientations and sizes. A 2D-Gabor filter is given by a Gaussian kernel function that is modulated by a complex sinusoidal wave:

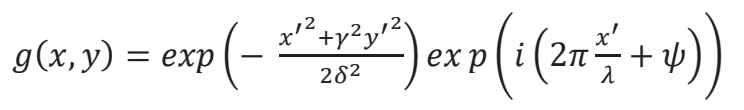

where $x^{\prime}=x \cos \theta+y \sin \theta, y^{\prime}=-x \sin \theta+y \cos \theta, \lambda$ represents the wavelength of the sinusoidal factor, $\theta$ is the orientation, $\psi$ is the phase offset, $\delta$ is the standard deviation of the Gaussian envelope and $\gamma$ is the spatial aspect ratio. The bandwidth of the Gabor filter $b$ is related to the ratio $\sigma / \lambda$. In our implementation, we have set the different parameters as follows: $\lambda \in\{4,6,8\}, \gamma \in\{0.25,0.5\}, \theta=\{\mathrm{k} \pi / 4, \mathrm{k}=0, \ldots$, $7\}$, and $b$ is set to 1 . We consider the obtained response with maximum modulus across all orientations (defined by $\theta$ ) for each pixel position and scale, which produce $3 \times 2 \times 1=6$ different configurations of Gabor filters. Fig. 4 (c) shows an example of Gabor filter application on one image from the DRIVE dataset.

\section{3) Gradient-based features}

Image gradient is an operator used in image processing to detect edges and extract texture features by computing the directional change in the intensity into an image. Equation (6) defines the gradient at each point of the image:

$$
\nabla f=\left[\begin{array}{l}
g_{x} \\
g_{y}
\end{array}\right]=\left[\begin{array}{l}
\frac{\partial f}{\partial x} \\
\frac{\partial f}{\partial y}
\end{array}\right]
$$

where: $\frac{\partial f}{\partial x}$ and $\frac{\partial f}{\partial y}$ are the gradient in the $\mathrm{x}$ and $\mathrm{y}$ directions, respectively. Usually the convolution of the image with such operators like the Sobel or the Prewitt operator is used to approximate the image gradient. In this work, we have used the Sobel operator:

$$
\begin{gathered}
G_{x}=\left[\begin{array}{ccc}
-1 & 0 & +1 \\
-2 & 0 & +2 \\
-1 & 0 & +1
\end{array}\right] * I \\
G_{y}=\left[\begin{array}{ccc}
-1 & -2 & -1 \\
0 & 0 & 0 \\
+1 & +2 & +1
\end{array}\right] * I
\end{gathered}
$$

And

where * denotes 2-D convolution, I respresents the source image. At each point in the image, the gradient magnitude $G$ and direction $\Theta$ are computed by combining $G_{x}$ and $G_{y}$ using the following equations:

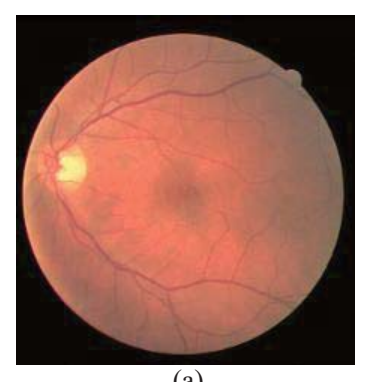

(a)

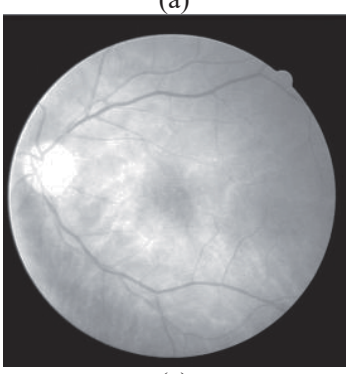

(c)

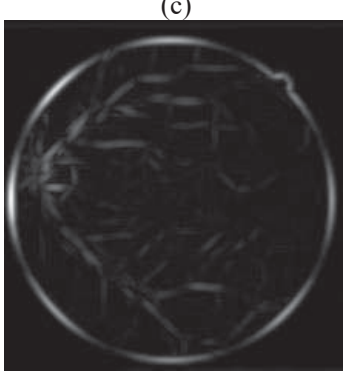

(e)

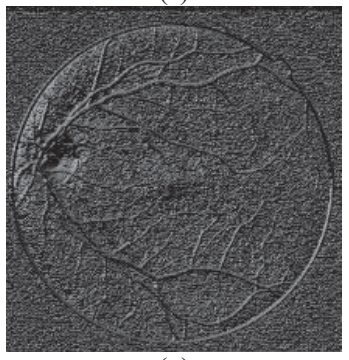

(g)

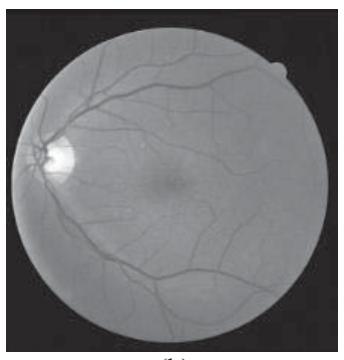

(b)

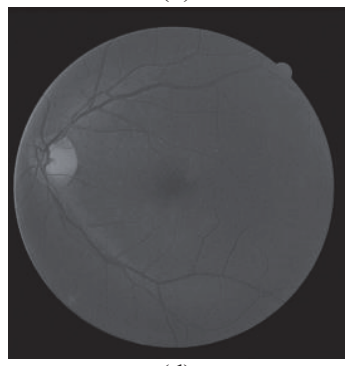

(d)

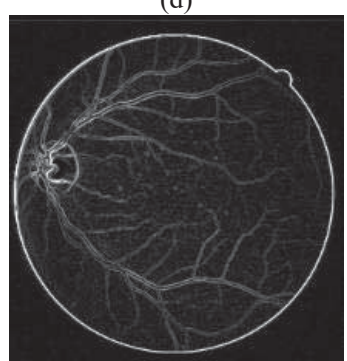

(f)
Fig. 4. Example of extracted features from the DRIVE dataset (a) original image, (b) Green plane (c) Red plane, (d) Blue plane, (e) Gabor filter response for $\lambda=8$ and $\gamma=0.5$, and (f) Gradient magnitude and (g) gradient direction.

$$
\begin{aligned}
& G=\sqrt{G_{x}^{2}+G_{y}^{2}} \\
& \Theta=\operatorname{arctg}\left(\frac{G_{y}}{G_{x}}\right) .
\end{aligned}
$$

An example of the gradient magnitude and direction for an image from the DRIVE dataset are illustrated in Fig. 4(d) and Fig. 4(e), respectively.

In this work, all extracted features are normalized between 0 and 1 using the following equation:

$$
U_{k}(i)=\frac{U_{k}(i)-\min \left(U_{k}\right)}{\max \left(U_{k}\right)-\min \left(U_{k}\right)}
$$


where $U=\left[U_{1}, U_{2}, \ldots, U_{n}\right]$, and each component $U_{k}$ is a vector which contains the values of a particular feature (e.g. average or standard deviation) of all the pixels of the image.

\section{Evaluation}

In order to thoroughly evaluate the proposed framework and compare its performance against related state-of-theart techniques, we have used the most common objective evaluation metrics based on accuracy, specificity and sensitivity:

$$
\begin{gathered}
\text { Accuracy }=\left(T_{P}+T_{N}\right) /\left(T_{P}+F_{P}+T_{N}+F_{N}\right) \\
\text { Specificity }=\mathrm{T}_{\mathrm{N}} /\left(\mathrm{T}_{\mathrm{N}}+\mathrm{F}_{\mathrm{P}}\right) \\
\text { Sensitivity }=\mathrm{T}_{\mathrm{P}} /\left(\mathrm{T}_{\mathrm{P}}+\mathrm{F}_{\mathrm{N}}\right)
\end{gathered}
$$

where TP represents the true positives, which are the vessel pixels detected as vessels, FP represents the false positives which represent non-vessel pixels detected as vessels, TN represents the true negatives which are the non-vessel pixels detected as non-vessels and FN is the false negatives which are the vessel pixels detected as non-vessels.

\section{ESN Setup}

We have randomly initialised the input and the reservoir weights between -1 and +1 . As described in Section III, the sigmoidal functions are a common choice of activation functions for the reservoir nodes. However, we have used the hyperbolic tangent function (commonly denoted by tanh). Additionally, the ESN input layer does not connect directly with the readout layer and the latter does not feedback to the former. The Echo State Network parameters are set according to the ESN design guidelines derived in our previous work [18] where the connectivity density, the spectral radius and the reservoir size are set $0.2,0.001$ and 100 , respectively. As discussed in the authors' previous work [18], it was demonstrated that small set of randomly selected neurons was sufficient enough for extracting good quality image features for producing adequate general-purpose image segmentation performance. Therefore, so in our experiments here we have selected randomly a small set of 20 nodes from the dynamic reservoir for use as the newly projected pixel features for classification. We have varied the number of selected neurons and extensive experiments were conducted before settling on 20 neurons which is found to be the minimum number of neurons sufficient for achieving competitive blood vessel segmentation performance.

\section{E. Readout Setup}

As discussed earlier, we have adopted an MLP to realise the ESN readout function of our ESN. It has been reported that mapping $\mathrm{x}(\mathrm{n})$ to $\mathrm{y}(\mathrm{n})$ using MLP as the ESN readout is theoretically more powerful than other linear readouts. In addition, MLPs are more suitable for learning non-linear mappings as discussed in [9]. In our experiments, the used MLP has two hidden layers of 25 sigmoidal neurons each, this choice is made through trial and error. The MLP output layer contains only one neuron with a linear activation function limited between 0 and 1 . Its desired output is set to 1 if the

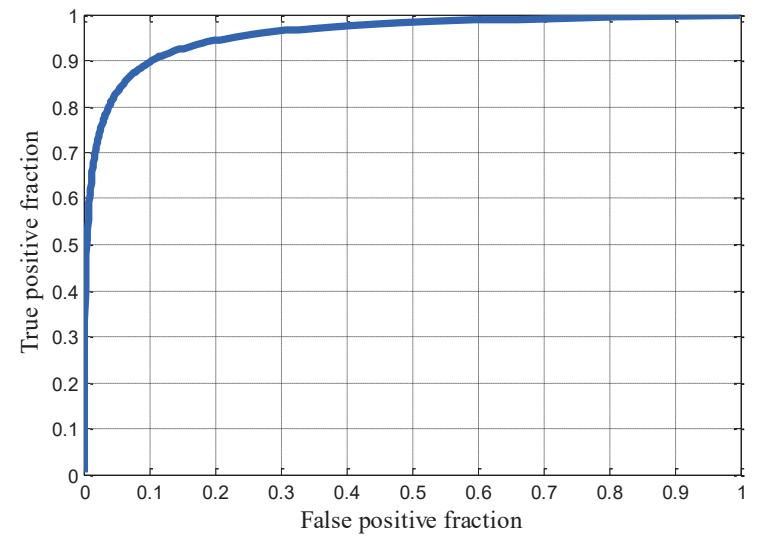

Fig. 5. ROC curve of the proposed framework for the DRIVE dataset.

input pixel belongs to the vessel class, otherwise (i.e. input pixel is non-vessel) it is set to 0 .

\section{F. Training}

As discussed in Section V.A, the DRIVE dataset contains 20 training images and 20 testing images. The training group contains 569415 vessel pixels in total and 3971591 background pixels (non-vessel). To train the MLP (which is used

to realise the ESN readout), we have randomly selected without replacement a total of 56941 vessel pixels and a similar number of non-vessel pixels, from the training images. In total, we have selected 113882 pixels which amounts to approximately $2.5 \%$ of the total number of pixels in the training images. After training, the 20 testing images are used to test the segmentation performance of the proposed ESN based framework.

\section{RESULTS AND DISCUSSION}

This section presents a comparison of the obtained performance results with the state-of-the-art methods [23], [29]-[33]. A threshold is used to classify a pixel as vessel or non-vessel (i.e background) and the Received Operating Characteristic (ROC) curve is then computed. Fig. 5 shows the ROC curve obtained by varying the decision threshold between 0 and 1 .

Table I shows a comparison of the obtained performance with respect to area under curve (AUC), accuracy (Acc), specificity $(\mathrm{Sp})$ and sensitivity (Se) against those reported by other related methods. For each method in Table I, the reported $\mathrm{Se}$ and $\mathrm{Sp}$ values correspond to the optimal decision threshold that gives the maximum accuracy (Acc). Overall, the proposed framework achieves comparable performance with the related state-of-the-art methods. For example, our method achieves an accuracy (Acc) of 0.9464 which is slightly higher than those of [29], [30] and [33] which achieved $0.9461,0.9452$ and 0.9441 , respectively, and slightly lower than [31], [32] which achieved 0.9480 and 0.9474 , respectively. However, Li et al. method [23] achieved 0.9527 and outperformed all of the other techniques. Regarding the AUC measure, the proposed framework 
TABLE I. COMPARATIVE EVALUATION OF THE OBTAINED SEgmentation PERFormance Using the Drive Dataset

\begin{tabular}{|l|c|c|c|c|c|}
\hline \multicolumn{1}{|c|}{ Method } & Year & Se & Sp & Acc & AUC \\
\hline Li [23] & 2016 & $\mathbf{0 . 7 5 6 9}$ & $\mathbf{0 . 9 8 1 6}$ & $\mathbf{0 . 9 5 2 7}$ & 0.9738 \\
\hline Fraz [31] & 2012 & 0.7406 & 0.9807 & 0.9480 & $\mathbf{0 . 9 7 4 7}$ \\
\hline Cheng [32] & 2014 & 0.7252 & 0.9798 & 0.9474 & 0.9648 \\
\hline Our proposed method & 2016 & 0.7173 & 0.9786 & 0.9464 & 0.9590 \\
\hline Soares [30] & 2006 & 0.7332 & 0.9782 & 0.9461 & 0.9614 \\
\hline Marin [33] & 2011 & 0.7067 & 0.9801 & 0.9452 & 0.9588 \\
\hline Staal [29] & 2004 & N.A & N.A & 0.9441 & 0.9520 \\
\hline
\end{tabular}

TABLE II. Average Processing TIME of One Retinal IMAGE BY DIFFERENT SEGMENTATION TECHNIQUES

\begin{tabular}{|l|l|}
\hline \multicolumn{1}{|c|}{ Method } & Processing time \\
\hline Li [23] & $1.2 \mathrm{~min}$ \\
\hline Fraz [31] & $2 \mathrm{~min}$ \\
\hline Soares [30] & $3 \mathrm{~min}$ \\
\hline Marin [33] & $1.5 \mathrm{~min}$ \\
\hline Staal [29] & $15 \mathrm{~min}$ \\
\hline Cheng [32] & Less than $1 \mathrm{~min}$ \\
\hline Our proposed method & $\mathbf{1 8}$ seconds \\
\hline
\end{tabular}

achieved 0.9590, which is higher than those of [29], [33] methods achieving 0.9580 and 0.9520 , respectively, and lower than those of [31], [23], [32] and [30] which achieved $0.9747,0.9738,0.9648$ and 0.9614 , respectively. Therefore, our method tends to obtain comparable segmentation accuracy result in comparison with the other state-of-the-art techniques

In addition, Fig. 6 shows sample segmentations obtained by the proposed ESN framework. It clearly shows that the segmentation results of our method are visually very similar to ground-truth segmentations.

As discussed in Section V.F, the percentage of training pixels used in our approach is approximatively $2.5 \%$ from the total number of pixels which form the training set, unlike the methods by $\mathrm{Li}$ [23] and Soares [30] which have respectively used $30 \%$ and $22 \%$ of the total pixels to train their classifiers. That is, a small amount of training pixels was found to be enough to train our approach and achieve comparable accuracy results with other methods.

Table II reports the obtained average processing time of one retinal image segmentation for the different techniques. The results of the other techniques are taken from [23]. The processing time of our technique involves three stages: the initial features extraction, the ESN reservoir output computation and the classification using the already trained MLP. These three stages take about 11,2 and 5 seconds, respectively. Therefore, our technique requires approximately 18 seconds to segment one image from the DRIVE dataset. MATLAB 2013a has been used to implement the proposed ESN-based framework on an Intel i7-2600 CPU (3.39 GHz, 4 GB RAM). Table II shows that our approach clearly outperforms all other techniques in terms of processing time of one retinal image. Usually the processing time of supervised methods involves the features extraction and the classification. However, some methods require preprocessing before extracting pixel features from images and as well as post-processing after computing the output for each pixel and classifying it as a vessel or background. For
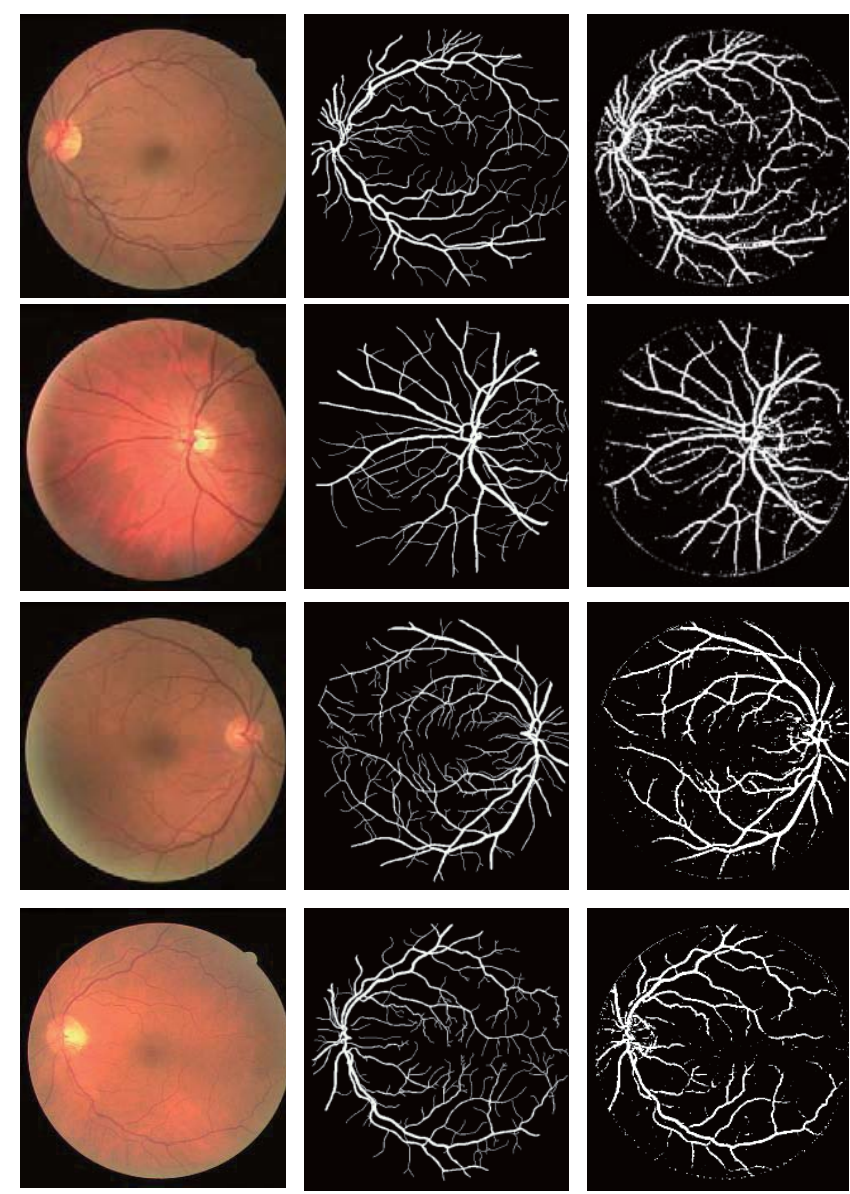

Fig. 6. Sample segmentations using the proposed ESN method. Left to right: original image, ground-truth segmentation, the proposed approach.

instance, Soares et al. [30] have developed an iterative algorithm which gets rid of the high contrast difference between the retinal fundus and the area outside the aperture. The pre-processing step of this method consists of iteratively growing a region of interest that is initially determined by the camera aperture. They firstly determine the pixels located on the exterior border of the initially identified region of interest. The value of each of these pixels is then replaced with the mean value of its neighbouring pixels within the identified region of interest which is expanded by including these modified pixels. This iterative process is considered as an artificial increase of the grown region of interest. This preprocessing procedure is time consuming. They also used a Gabor wavelet transform for 18 different orientations. However, in our work we use Gabor filter for only 8 different orientations. Staal et al. [29] computed a group of 27 features for each pixel using a ridge-based vessel detection method. They then applied a sequential forward selection method to extract features with the best class separability and used k$\mathrm{NN}$ to classify them. The computation of such number of features and the scheme of best parameters selection are time consuming. In Marin et al. [33], a series of pre-processing operations are applied before extracting the pixels features. It consists of the following three stages: (1) removal of the reflex of the vessel central light, homogenization of the image 
background and, finally, enhancement of the segmented vessel areas. The aim of the first stage is to remove the central lighting of the blood vessel. For this reason, they filter the retinal image by applying a morphological opening. This operation has the risk to remove thin vessels. The second stage is to homogenize the background in order to remove its lightening variations. The background is estimated from the image through the application of filtering using a large arithmetic mean kernel. Then the estimated background is subtracted from the resulted image of the first stage. The final pre-processing stage consists in the enhancement of the generated vessel-image which is achieved through the estimation of the complementary of the homogenized image followed by the application of the morphological Top-Hat transformation. These various pre-processing operations take considerable time. Unlike these methods, neither preprocessing nor post- processing are used in the proposed ESN based segmentation approach.

\section{CONCLUSION}

We have proposed an ESN-based framework for blood vessel segmentation in retinal fundus images. In this study, we have studied the viability of using the ESN reservoir for extracting more expressive pixel features which can be used for achieving competitive blood vessel segmentation in retinal images. It was found that while the proposed technique achieved comparable accuracy performance with state-ofthe-art vessel segmentation techniques, it clearly outperformed all of the other techniques in terms of processing time required to segment a retinal image. Further work is planned to investigate the use of other classifiers as the ESN readout, and the use of the Liquid State Machine (LSM) which is similar to the ESN concept but uses more biologically plausible spiking neuron models as reservoir nodes instead of the classical rate-based neurons used in the ESN based technique.

\section{REFERENCES}

[1] M. M. Fraz, P. Remagnino, A. Hoppe, B. Uyyanonvara, A.R. Rudnicka, C.G. Owen, and S.A. Barman, "Blood vessel segmentation methodologies in retinal images-A survey," Comput. Methods Progr. Biomed., vol. 108, pp. 407-433, Oct. 2012.

[2] A. A. H. A. R. Youssif, A. Z. Ghalwash, and A. R. Ghoneim, "Optic disc detection from normalized digital fundus images by means of a vessels' direction matched filter," IEEE Trans. Med. Imag., vol. 27, no. 1, pp. 11-18, Jan. 2008.

[3] G. K. Matsopoulos, P. A. Asvestas, N. A. Mouravliansky, and K. K. Delibasis, "Multimodal registration of retinal images using self organizing maps," IEEE Trans. Med. Imag., vol. 23, no. 12, pp. 15571563, Dec. 2004.

[4] C. Marino, M. G. Penedo, M. Penas, M. J. Carreira, and F. onzalez, "Personal authentication using digital retinal images," Pattern Anal. Appl., vol. 9, pp. 21-33, May 2006.

[5] S. Chaudhuri, S. Chatterjee, N. Katz, M. Nelson, and M. Goldbaum, "Detection of blood vessels in retinal images using twodimensional matched filters," IEEE Trans. Med. Imag., vol. 8, no. 3, pp. 263-269, Sep. 1989.

[6] K. S. Sreejini and V. K Govindan, "A Review of Computer Aided Detection of Anatomical Structures and Lesions of DR from Color Retina Images," I.J. Image, Graphics and Signal Processing, vol. 11, pp. 55-69, 2015.
[7] M. Garg and S. Gupta, "Retinal Blood Vessel Segmentation Algorithms: A Comparative Survey," International Journal of BioScience and Bio-Technology, vol. 8, no. 3, pp. 63-76, 2016.

[8] N. Gupta and E. Aarti, "A Review on Segmentation Techniques for Extracting Blood Vessels from Retina Images," International Journal of Emerging Research in Management \&Technology, vol. 5, March 2016.

[9] M. Lukosevicius and H. Jaeger, " Reservoir computing approaches to recurrent neural network training," Computer Science Review, vol. 3, pp. 127-149, 2009.

[10] D. Verstraeten, B. Schrauwen, M. D'Haene and D. Stroobandt, "An experimental unification of reservoir computing methods," Neural Netw., vol. 20, pp. 391-403, 2007.

[11] H. Jaeger, "The echo state approach to analyzing and training recurrent neural networks," Technical Report, vol. 148, Bonn, Germany: German National Research Center for Information Technology (GMD), pp. 147, 2001.

[12] W. Maass, T. Natschläger and H. Markram, " Real-time computing without stable states: A new framework for neural computation based on perturbations," Neural Comput., vol. 14, pp. 2531-2560, 2002.

[13] S. Lun, X. Yao, H. Qi, and H. Hu, "An effective multivariate time series classification approach using echo state network and adaptive differential evolution algorithm," Expert Syst. Appl., vol. 43, pp. 237249, January 2016.

[14] M. Chen, W. Saad, and C. Yin, "Optimized uplink-downlink decoupling in lte-u networks: An echo state approach," in IEEE International Conference on Communications, Mobile and Wireless Networks Symposium, pp. 1-6, May 2016.

[15] D . Suganthi and S. Purushothaman, "FMRI segmentation using echo state neural network," International Journal of Image Processing, vol. 2, pp. 1-9, 2008.

[16] P. Koprinkova-Hristova, D. Angelova, D. Borisova and G. Jelev, "Clustering of spectral images using Echo state networks," IEEE International Symposium on Innovations in Intelligent Systems and Applications, ’pp. 1-5, Jun. 19-21, 2013.

[17] B. Meftah, O. Lezoray and A. Benyettou, "Novel approach using echo state networks for microscopic cellular image segmentation," Cogn. Comput., pp. 1-9, 2015.

[18] A. Souahlia, A. Belatreche, A. Benyettou, and K, Curran "An experimental evaluation of echo state network for colour image segmentation,", IEEE International Joint Conference on Neural Networks, Jul. 24-29, 2016.

[19] B. Zhang, L. Zhang, L. Zhang, and F. Karray, "Retinal vessel extraction by matched filter with first-order derivative of gaussian," Comput. Biol. Med., vol. 40, no. 4, pp. 438-445, 2010.

[20] G. Azzopardi, N. Strisciuglio, M. Vento, and N. Petkov, "Trainable COSFIRE filters for vessel delineation with application to retinal mages," Med. Image Anal., vol. 19, pp. 46-57, Feb. 2015.

[21] F. K. H. Quek and C. Kirbas, "Vessel extraction in medical images by wave-propagation and traceback," IEEE Trans. Med. Imaging, vol. 20, no. 2, pp. 117-131, Feb. 2001.

[22] X. Gao, A. Bharath, A. Stanton, A. Hughes, N. Chapman, and S. Thom, "A method of vessel tracking for vessel diameter measurement on retinal images," in Proc. ICIP'01, pp. II: 881-884, 2001.

[23] Q. Li, B. Feng, L. Xie, P. Liang, H. Zhang, and T. Wang, "A CrossModality Learning Approach for Vessel Segmentation in Retinal Images", IEEE Trans. Med. Imaging, vol. 35, no. 1, pp.109-118, January 2016.

[24] B. S. Y. Lam and H. Yan, "A novel vessel segmentation algorithm for pathological retina images based on the divergence of vector fields," IEEE Trans. Med. Imag., vol. 27, no. 2, pp. 237-246, Feb. 2008.

[25] L. Espona, M. J. Carreira, M. G. Penedo, and M. Ortega, "Retinal vessel tree segmentation using a deformable contour model," in Proc. 19th Int. Conf. Pattern Recognit, pp. 2128-2131, 2008.

[26] L. Gang, O. Chutatape, and S. M. Krishnan, "Detection and measurement of retinal vessels, in fundus images using amplitude 
modified second-order Gaussian filter," IEEE Trans. Biomed. Eng., vol. 49, no. 2, pp. 168-172, Feb. 2002.

[27] L. Espona, M. J. Carreira, M. Ortega, and M. G. Penedo, "A snake for retinal vessel segmentation," Pattern Recognition and Image Analysis, vol. 4478, pp. 178-185, 2007.

[28] M. Niemeijer, J. Staal, B. van Ginneken, M. Loog, and M. D. Abramoff, "Comparative study of retinal vessel segmentation methods on a new publicly available database," Med. Imag. 2004: Image Process., vol. 5370, pp. 648-656, 2004.

[29] J. Staal, M. D. Abramoff, M. Niemeijer, M. A. Viergever, and B. van Ginneken, "Ridge-based vessel segmentation in color images of the retina," IEEE Trans. Med. Imag., vol. 23, no. 4, pp. 501-509, Apr. 2004

[30] J. V. B. Soares, J. J. G. Leandro, R. M. Cesar, H. F. Jelinek, and M. J. Cree, ""Retinal vessel segmentation using the 2-D Gabor wavelet and supervised classification," IEEE Trans. Med. Imag., vol. 25, pp. 1214 1222, Sep. 2006.

[31] M. M. Fraz, P. Remagnino, A. Hoppe, B. Uyyanonvara, A.R. Rudnicka, C.G. Owen, and S.A. Barman, "An ensemble classification-based approach applied to retinal blood vessel segmentation," IEEE Trans. Biomed. Eng., vol. 59, no. 9, pp. 2538-2548, Sep. 2012.

[32] E. K. Cheng, L Du, Y Wu, Y, J, Zhu V, Megalooikonomou, and H, Ling, "Discriminative vessel segmentation in retinal images by fusing context-aware hybrid features," Mach. Vis. Appl., vol. 25, pp. 1779 1792, Oct. 2014.

[33] D. Marin, A. Aquino, M. E. Gegundez-Arias, and J. M. Bravo, "A new supervised method for blood vessel segmentation in retinal images by using gray-level and moment invariants-based features," IEEE Trans. Med. Imag., vol. 30, no. 6, pp. 146-158, Jan. 2011. 